\title{
DESIGN FOR CONNECTING SPATIAL DATA INFRASTRUCTURES WITH SENSOR WEB (SENSDI)
}

\author{
D. Bhattacharya*, M. Painho \\ 1Nova Information Management School, University Nova de Lisboa, Lisbon, Portugal. \\ dbhattacharya@novaims.unl.pt \\ painho@novaims.unl.pt
}

ISPRS - WG IV/4 - Geospatial Data Infrastructure- Full Papers

\begin{abstract}
KEY WORDS: Sensor web, spatial data infrastructures, application programming interface, web enabled architecture, semantic integration.
\end{abstract}

\begin{abstract}
:
Integrating Sensor Web With Spatial Data Infrastructures (SENSDI) aims to extend SDIs with sensor web enablement, converging geospatial and built infrastructure, and implement test cases with sensor data and SDI. It is about research to harness the sensed environment by utilizing domain specific sensor data to create a generalized sensor webframework. The challenges being semantic enablement for Spatial Data Infrastructures, and connecting the interfaces of SDI with interfaces of Sensor Web. The proposed research plan is to Identify sensor data sources, Setup an open source SDI, Match the APIs and functions between Sensor Web and SDI, and Case studies like hazard applications, urban applications etc. We take up co-operative development of SDI best practices to enable a new realm of a location enabled and semantically enriched World Wide Web - the "Geospatial Web" or "Geosemantic Web" by setting up one to one correspondence between WMS, WFS, WCS, Metadata and 'Sensor Observation Service' (SOS); 'Sensor Planning Service' (SPS); 'Sensor Alert Service' (SAS); a service that facilitates asynchronous message interchange between users and services, and between two OGC-SWE services, called the 'Web Notification Service' (WNS). Hence in conclusion, it is of importance to geospatial studies to integrate SDI with Sensor Web. The integration can be done through merging the common OGC interfaces of SDI and Sensor Web. Multi-usability studies to validate integration has to be undertaken as future research.
\end{abstract}

\section{Introduction}

Location is everywhere and "everywhen". For centuries, philosophers and academicians have understood that humans are spatial/temporal beings. Everything we do happens somewhere and "somewhen" [1-2]. Almost every decision that an individual or organization makes has some geospatial component. Almost any piece of information stored in a database has a location attribute. We are fast approaching the technical means to track, measure, capture, analyze, and database the location element for human and other activity, including the movements of any individual or object in space. There are at once both huge benefits and serious implications associated with such a technical capability. Many SDI projects are faced with developing policies and approaches to dealing with maximizing the benefits and minimizing the impacts of potential wastefulness [3-5]. The technology infrastructure, the geospatially enabled internet and Web, enables the ability to capture, communicate and process location based information, and it also provides tools and technologies to prevent its abuse. This paper documents some of the geospatial standards that are available for use in any SDI implementation. The research is about how geospatial standards can work in concert with other IT standards to support rights management, security, privacy, authentication, and so forth [6-8].

The state of the art in this area are some ongoing activities that are defining architectures and best practices for integrating sensor networks and observed data into existing and new SDI applications. SANY (Sensor Systems Anywhere) is an example [9-11] describing a possible concept. The SANY Framework Programme 6 (FP6) integrated project focuses on interoperability of in-situ sensors and sensor networks.

The SANY architecture will provide a quick and cost-efficient way to reuse of data and services

\footnotetext{
* Corresponding author
} 
from currently incompatible sensor- and datasources. The SANY activity will deliver:

- Standard service-oriented architecture for environmental sensor networks. SANY services shall be self-describing and capable of seamless "plug and measure" and sharing ("virtual networks").

- Reference implementations of re-usable sensor- and domain-agnostic services, including decision support and generalized data fusion services.

- Three innovative risk management applications covering the areas of air pollution, marine risks and geo hazards. Some other activities in progress are [12-15]. But these projects are mostly into architecture phase not thinking in terms of a broad scaled SDI and Sensor Web. The application phase is still far-off. SENSDI can have a real edge over the currently under development projects of similar kind in terms of impact on urban development, hazard management and mitigation, digital mapping etc [16-19].

\subsection{Research Gaps:}

- Traditional data publishers, mapping agencies, cadastral agencies, which are used to serving a professional market in a particular way, have largely developed the SDIs thereby short-sighting it.

- Limited in taking hints from the broader web i.e. internet.

- Vision of a truly location based "geospatial web".

- Importance of location at all levels of the internet stack.

- New best practices are emerging and rapidly evolving government, businesses, academia and citizens.

- SDI community is in position to reap significant benefits from new developments as well as driving the maturation of the Geospatial Web.

\section{Main Goals}

- Extend SDIs with sensor web enablement.
- Converging geospatial and built infrastructure.

- Implement test cases with sensor data and SDI.

- Harnessing the sensed environment.

- Utilizing domain specific sensor data.

- Semantic Enablement for Spatial Data Infrastructures.

- Connecting the interfaces of SDI with interfaces of Sensor Web.

\subsection{Description:}

The concept of the "sensor web" is a type of sensor network that is especially well suited for environmental monitoring. The phrase "sensor web" is also associated with a sensing system which heavily utilizes the World Wide Web [2022]. OGC's Sensor Web Enablement (SWE) framework defines a suite of web service interfaces and communication protocols abstracting from the heterogeneity of sensor (network) communication. A spatial data infrastructure (SDI) is a framework of spatial data, metadata, users and tools that are interactively connected in order to use spatial data in an efficient and flexible way [23-25]. It's the development and implementation of a pervasive database to store and exchange geospatial data for governance and other users in the civic sector. It is envisioned that within 8 to 10 years, the vast majority of all geospatial content will be from sensor networks and systems. The volume of available sensed content will be orders of magnitude more than it is today. Hence a consistent set of encoding and interface standards as shown in Fig. 1 would have to be mandatorily developed for adapting and integrating sensor networks into an SDI application and OGC's Sensor Web Enablement (SWE) activity [26-29].

2.2 Problems that will be addressed:

- Identify readily useable sensor data sources / sensor web architecture.

- Setup an open source SDI.

- Match the APIs and functions between Sensor Web and SDI.

- Case studies like hazard applications, urban applications etc. 
2.3 Methodology to be used:

2.3.1 The concepts of Sensor Web could be understood as:

- a service for retrieving sensor 'observation' data and meta-information, the so-called 'Sensor Observation Service' (SOS);

- a service for sensor planning and executing tasks, called the 'Sensor Planning Service' (SPS);

- a service that allows users to subscribe to specific alert types, known as the 'Sensor Alert Service' (SAS); a

- a service that facilitates asynchronous message interchange between users and services, and between two OGC-SWE services, called the 'Web Notification Service' (WNS).

\subsubsection{And that of SDI:}

Web Map Service (OGC \& ISO)

Style Layer Descriptor (OGC)

Feature Model \& GML (OGC \& ISO)

Web Feature Service (OGC \& ISO)

Web Coverage Service (OGC)

Web Map Context (OGC)

Catalogue (OGC)

Metadata (ISO 19115 \& OGC)

Others...

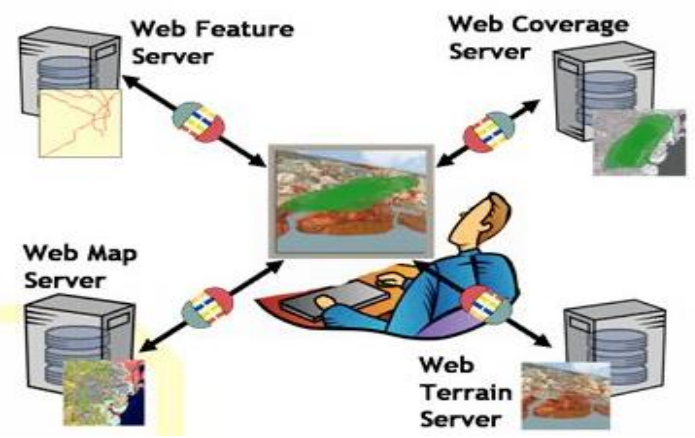

Fig. 1 : APIs of SDI that integrate to outside frameworks.

It is necessary to have a one-to-one correspondence between the elements mentioned in 2.3.1 and 2.3.2. A possible environment for establishing such interfaces could be GeoServer and GeoNode which provide an OGC compatible data store that can speak WMS, WFS, WCS and others in common formats like GML, GeoJSON, KML and GeoTiff [30-33].
Pointed out in Fig. 2 it can be connected to different spatial backends including PostGIS, Oracle Spatial, ArcSDE and others. The Catalog: GeoNetwork: GeoNetwork provides a standard catalog and search interface based on OGC standards. It is used via the CSW interface to create and update records when they are accessed in GeoNode. The Map Composer: GeoNode Client : The main map interface for GeoNode is the Map Composer / Editor. It talks to the other components via HTTP and JSON as well as standard OGC services [34-36].

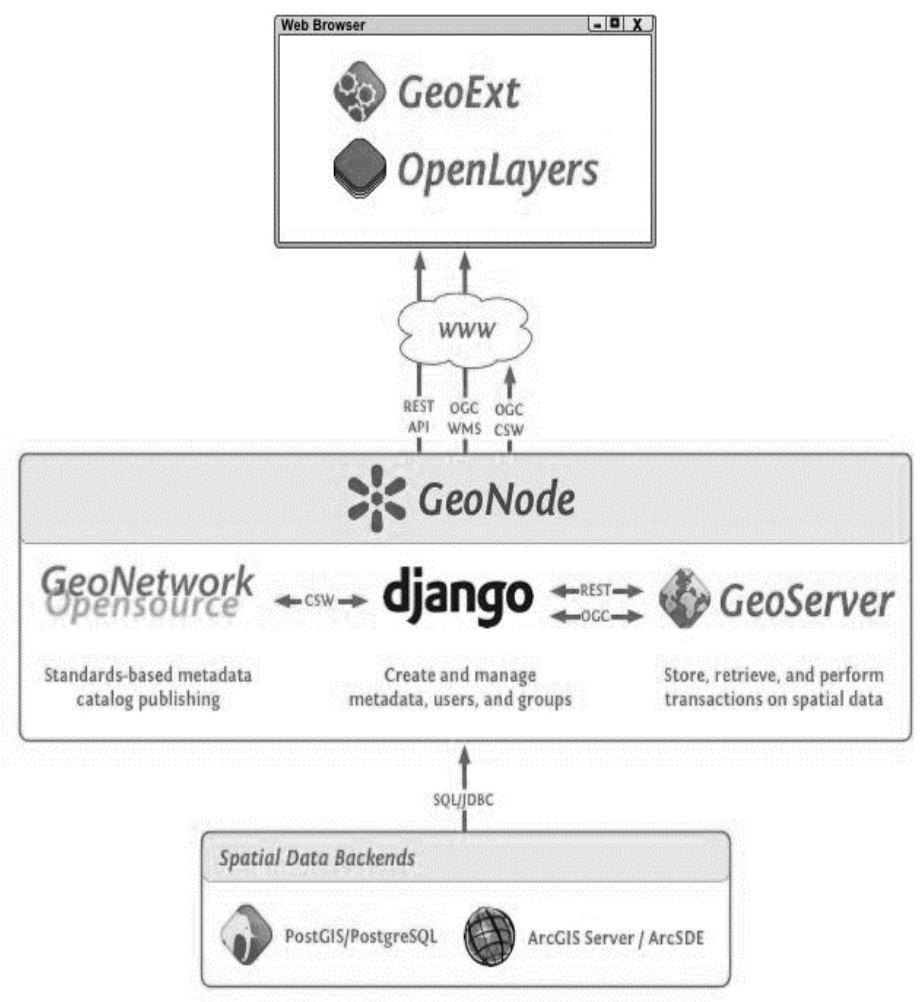

Fig. 2 : Top-down development structure for open access.

\section{METHODOLOGY}

SDI advancements coupled with continued advancements of Information and Communication Technology (ICT) and related standards are carrying us toward a "next level" of capability. What started as cooperative development of SDI best practices is evolving to enable a new realm of a location enabled and semantically enriched World Wide Web - some refer to as the "Geospatial Web" or 
"Geosemantic Web". The SDI community is in the position to reap significant benefits from new developments as well as driving the maturation of the Geospatial Web [36-38].

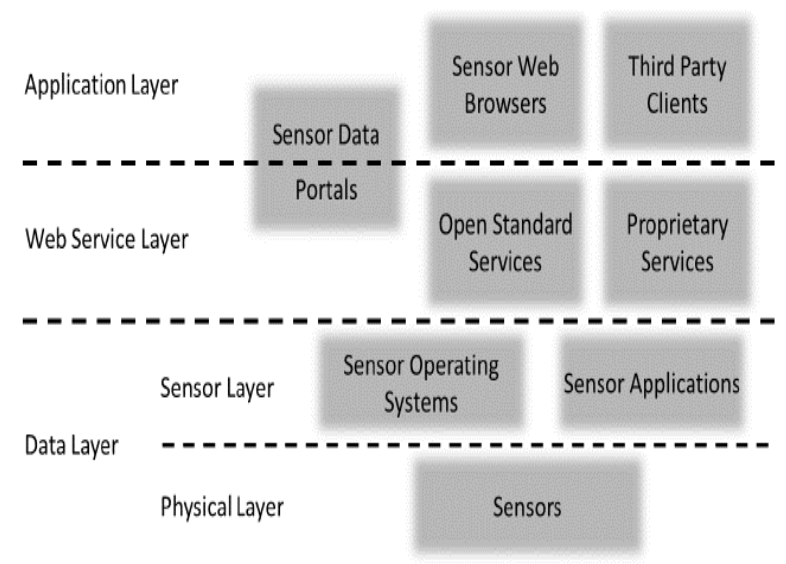

Fig. 3 : APIs from Sensor Web that enable integration with outside frameworks.

Critical decision making relies not only on the ability to fuse and apply core geospatial information, but also the ability to discover, access and apply real time information from sensors and sensor networks as shown in Fig. 3. A plethora of information about the quality of our land, air and water, weather, climate and natural and man-made risks can be harnessed by seamless and rapid access to sensors. In addition, sensors are critical components of building, transportation, utility and industry infrastructure. The ability to harness and render this information in a location context is a major challenge. Until recently though, there were no facilitating standards to make it easier to discover, access and integrate this information. The concept of plug-ins to interact amongst themselves from one framework to another makes the integration of SDI and sensor web possible (Fig. 4a and b). The web enablement is shown in Fig. 4 a where the architecture to capture geospatial elements and transmitting over the web is taken care of by webGIS standards and the open interfaces are utilized for latching on to the sensor network through a set of GML Clients as shown in Fig. $4 \mathrm{~b}$. Therefore, a consistent set of encoding and interface standards are mandatory for adapting and integrating sensor networks into an SDI application [21-25, 35-38].

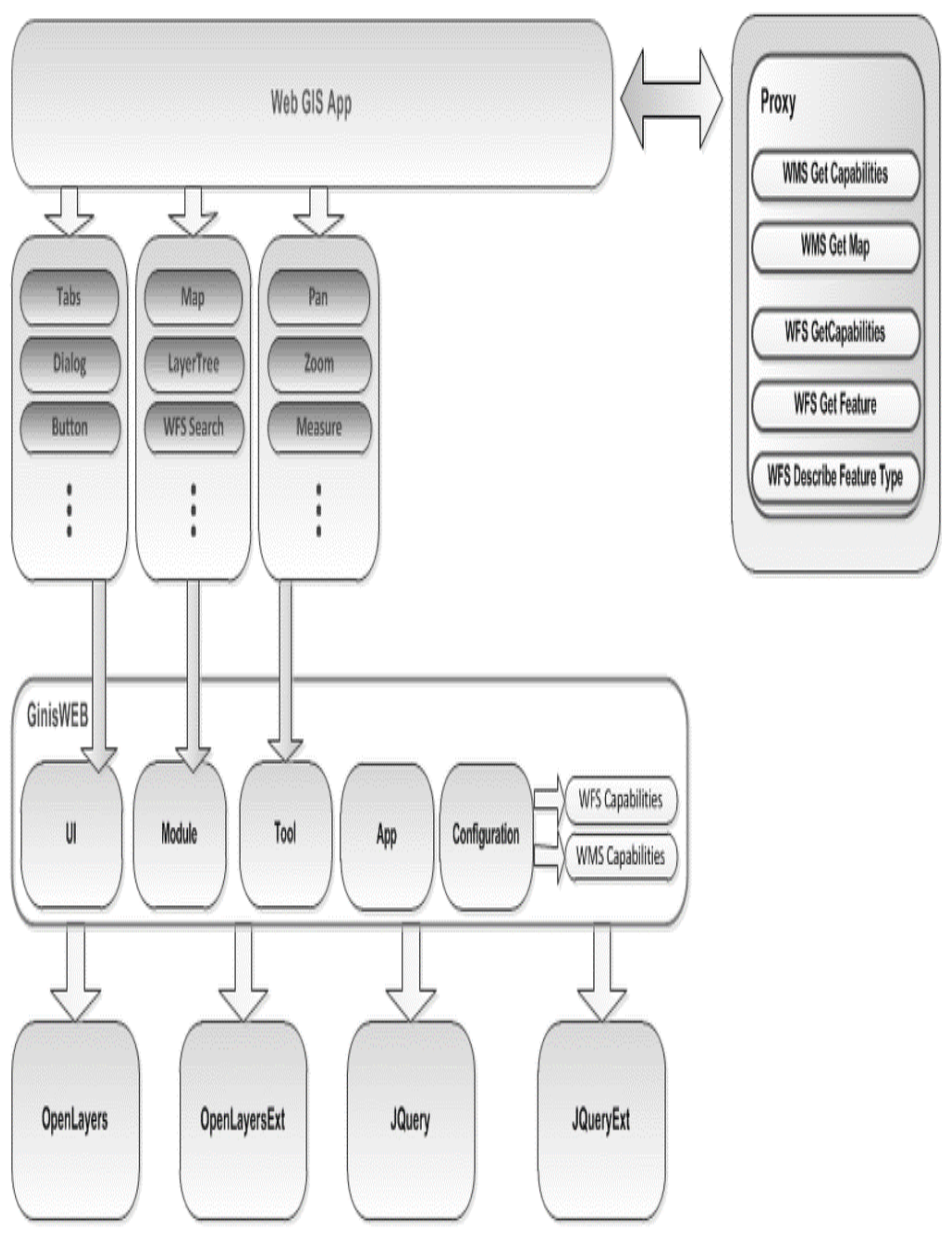

Fig. 4a : Flowchart of internal dataflow merged with sensor web and webGIS.

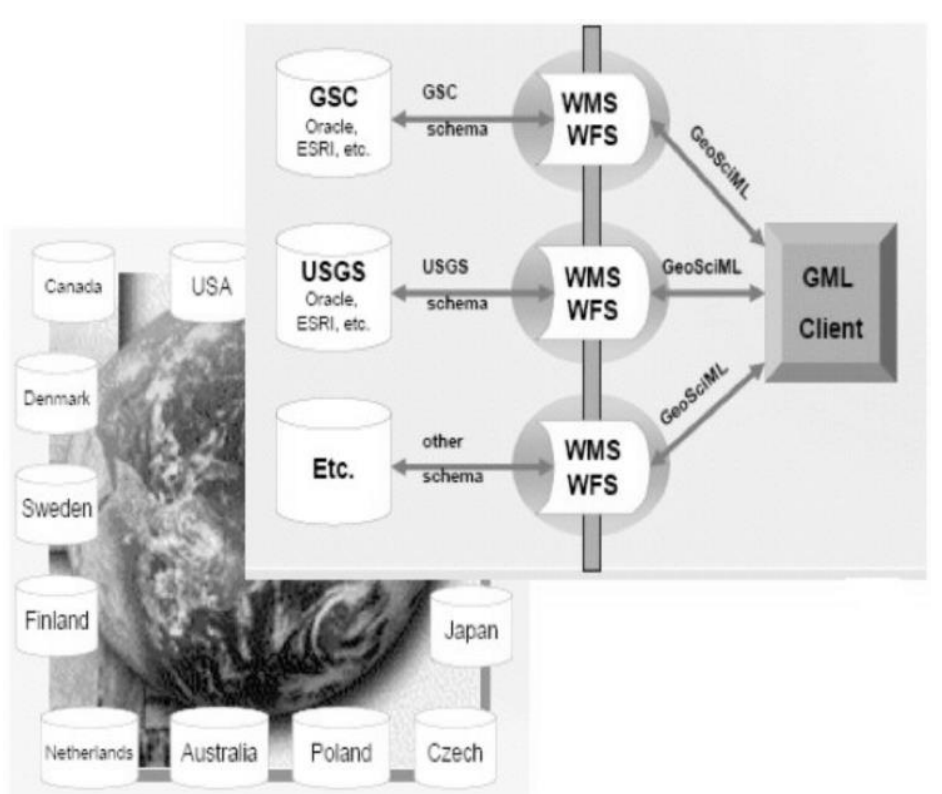

Fig. 4b : Sample integrated sensored webGIS GeoSciML is being worked on by EU agencies. 
Based upon the above methodologies, the development of critical structures could be possible that would help address the geospatial domain in the years to come.

i) Development of geosemantics for sensor-web and global SDIs.

ii) Development of interfaces for sensor-web and SDIs

iii) Application of integrated SENSDI to natural hazard mitigation.

iv) Application of SENSDI for smarter cities initiative.

v) Automated Real-Time System for Geospatial data processing in SenSDI.

\section{CONCLUSIONS}

SENSDI addresses smarter living conditions for citizens and better management of resources for administrators and industries by spatially enabling the new data sources coming up. Currently the vast capacity of spatially referencing newer sources of data and information is not being done. SDI concept has hit a roadblock towards further development. One very smart and efficient way forward is to integrate SDI with new data sources sensors that are coming up and creating a sensor web of spatially oriented information.

The results are to be published in reputed open journals and international conferences. The methodologies employ open source techniques. Open licensing is the way to future development.

The project is data intensive so interactions with data providers and sources is prominent. There must be meaningful interactions. The results of data processing and infrastructure building will be of interest to current and future stakeholders so that has to be formulated according to the university rules.

Expected users will be scientists developing systems like EU INSPIRE directives, OGC, ISO, public users and other agencies.
Very attractive market and research \& academic scope in spatial developments. Applicable to virtually all activities of commerce and governance.

SensorWeb and SDI are going to keep expanding in the next decade. It is envisioned that sensors are going to be so ubiquitous that like the WWW the addition of vast amount of sensors will keep happening like new data sources of present internet. The concept of SENSDI has to keep evolving step by step in the years to come to help overall development of a nation.

- Defense application of analyzing different sensor data.

- Health applications of medical sensor data.

- Climate change studies globally.

- Open publications.

- Open City Day SENSDI demonstrations for public.

- Online documentations and guidelines.

\section{REFERENCES}

[1] From Spatial Data Infrastructure to the Geospatial Web: Advancing the SDI Vision in a Changing Technology Environment http://www.gsdi.org/gsdiconf/gsdi10/papers/PS 5.2paper.pdf

[2] GeoCENS: A Geospatial Cyberinfrastructure for the World-Wide Sensor Web http://www.mdpi.com/1424-8220/13/10/13402 [3] SANY http://ercimnews.ercim.eu/en76/special/sensors-anywheresensor-web-enablement-in-risk-managementapplications

[4] Semantic Enablement for Spatial Data Infrastructures http://carsten.io/tgis09_sel.pdf [5] A. Weiser and A. Zipf. Web Service Orchestration of OGC Web Services for Disaster Management. LN in Geoinformation and Cartography, pages 239\{ 254. Springer, 2007. 
[6] Mayer c., Zipf A., (2009) "Integration and Visualization of dynamic Sensor Data into 3D Spatial Data Infrastructures in a standardized way", Paper presented at GeoViz 2009 Contribution of geovisualization to the concept of the digital city, Workshop, Hamburg, Germany, 05/03/2009, http://koenigstuhl.geog.uni-

heidelberg.de/publications/bonn/conference/geo Viz09.gdi3d.sensor.mayer_zipf.pdf

[7] Krzysztof Janowicz, Sven Schade, Arne Bröring, Carsten Keßler, Patrick Maué, and Christoph Stasch (2010) Semantic Enablement for Spatial Data Infrastructures. Transactions in GIS 14(2): 111-129. DOI:10.1111/j.14679671.2010.01186.x

[8] Neubauer, S. and Zipf, A. 2007. "Suggestions for Extending the OGC Styled Layer Descriptor (SLD) Specification into 3D - Towards Visualization Rules for 3D City Models," In: Urban DataManagement Symposium (UDMS 2007). Stuttgart, Germany.

[9] Basanow, J., Neis, P., Neubauer, S., Schilling, A. and Zipf, A. 2007. "Towards 3D Spatial Data Infrastructures (3D-SDI) based on Open Standards - experiences, results and future issues," In: Workshop on 3D Geo-Information (3D GeoInfo07). Delft, Netherlands.

[10] Schut, P. 2007. "OpenGIS Web Processing Service," Version 1.0.0. OGC 05-007r7.

[11] Botts, M. 2006. "OGC White Paper - OGC Sensor Web Enablement: Overview and High Level Architecture," Version 2.0. OGC 06$050 \mathrm{r} 2$.

[12] Mabrouk, M. 2005. "OpenGIS Location Services (OpenLS): Core Services," Version 1.1. OGC 05-016.

[13] Zipf, A. 2008. "Integrating 3D, Processing and Location Services into future SDIs," Tenth International Conference for Spatial Data Infrastructure. Port of Spain, Trinidad.

[14] Na, A. and Priest, M. 2007. "Sensor Observation Service," Version 1.0 OGC 06009r6.

[15] Stollberg, B. and Zipf, A. 2007. "OGC Web Processing Service Interface for Web Service Orchestration - Aggregating Geoprocessing Services in a Bomb Threat Scenario," In: Web and Wireless Geographical Information Systems (W2GIS 2007). Cardiff, UK.
[16] Friis-Christensen, A., Lutz, M., Bernard, L. and Ostländer, N. 2007. "Designing Service Architectures for Distributed Geoprocessing Challenges and Future Directions," Transactions in GIS, 11(6): 799-818.

[17] Foerster, T. and Stoter, J. 2006. "Establishing an OGC web processing service for generalization processes," Workshop of the ICA Commission on Map Generalisation and MultipleRepresentation, Portland, USA.

[18] Díaz, L., Granell, C. and Gould, M. 2008 in press. "Case Study:Geospatial processing services for "Case web-based hydrologicalapplications," In: J.T. Sample, K. Shaw, S. Tu and M. Abdelguerfi(Eds.): Geospatial Services and Applications for the Internet.Springer, 2008 (in press).

[19] Stollberg, B. and Zipf, A. 2008. "Geoprocessing Services for Spatial Decision Support in the Domain of Housing MarketAnalyses -Experiences from Applying the OGC Web Processing ServiceInterface in Practice," The 11th AGILE 2008 Conference on GIScience. Girona, Spain.

[20] Graul, C. and Zipf, A. 2008. "Biogeographic Research on the Spatial Web: Towards Interoperable Analysis Tools for Global Change Based on the Web Processing Service (WPS)," Digital Earth Summit on Geoinformatics. Potsdam, Germany.

[21] Gerlach, R., Schmullius, C. and Nativi, S. 2008. "Establishing a web processing service for online analysis of earth observation time series data," Geophysical Research Abstracts 10.

[22] Nash, E., Korduan, P. and Bill, R. 2007. "Optimising data flows in precision agriculture using open geospatial web services," In Stafford,J. V. (ed.): Precision Agriculture '07: Proceedings of the 6th EuropeanConference on Precision Agriculture, Wageningen Academic Publishers, pp. 753-759.

[23] Göbel, R. and Zipf, A. 2008. "How to define 3D Geoprocessing Operations for the OGC Web Processing Service. (WPS)? Towards a Classification of 3D Operations," International Workshop on Computational Geoinformatics. Perugia, Italy.

[24] Botts M. (Ed.) 2006. OpenGIS sensor model language implementation specification (Version 1.0). In: Open Geospatial Consortium, OGC, 
Document Number:05-086r2, Wayland, MA, USA, $117 p$.

[25] Chen, A., Di, L., Wei, Y., Liu, Y., Bai, Y., $\mathrm{Hu}$, C., Mehrotra, P., 2005. Grid enabled geospatial catalogue web services. In: Proc. American Society for Photogrammetry and Remote Sensing, ASPRS, Annual Conference, Baltimore, USA, 711 March, 10p.

[26] Cox S. (Ed.) 2006. OGCTM Observation and measurement (Version 0.13.0). In: Open Geospatial Consortium, OGC, Document Number: 05-087r3, Wayland, MA, USA, 136p.

[27] Di, L., 2007. Geospatial sensor web and self-adaptive earth predictive systems, SEPS. http://esto.nasa.gov/sensorwebmeeting/Papers/ Di.pdf (accessed 21.07.08).

[28] Delin, K., Jackson, S., 2001. The Sensor Web: A new instrument concept. In: Proc. The International Society for Optical Engineering (SPIE)'s Symposium on Integrated Optics, San Jose, CA, USA, 2425 January, 9p.

[29] Evans, J.D., 2003. OGCTM Web coverage service specification (Version 1.0.0). In: Open Geospatial Consortium, OGC, Document Number: 03-065r6, Wayland, MA, USA, 67p.

[30] Lee, E., Kim, M., Kim, M., Joo, I., 2005. A web services framework for integrated Geospatial coverage data. Lecture Notes in Computer Science 3481, 1136-1145.

[31] Lee, K.B., Reichardt, M.E., 2005. Open standards for homeland security sensor networks Sensor interconnection and integration through Web access. Institute of Electrical and Electronics Engineers (IEEE) Instrumentation and Measurement Magazine 8 (5), 14-21.

[32] Liang, SHL., Croitoru, A., Tao, CV., 2005. A distributed geospatial infrastructure for Sensor Web. Computer and Geoscience 31 (2), 221-231. [33] Meier, W., 2003. eXist: An open source native XML database. Lecture Notes in Computer Science 2593, 169-183.

[34] Na A., Priest M. (Eds.) 2006. OpenGIS sensor observation service implementation specification (Version 0.1.5). In: Open Geospatial Consortium, OGC, Document Number: 06-009r1, Wayland, MA, USA, 187p. [35] T Usländer, $\mathrm{P}$ Jacques, I Simonis, K Watson, Designing environmental software applications based upon an open sensor service architecture, Environmental Modelling \& Software, 25, (2010), 977-987.

[36] A Kansal, F Zhao, Location and Mobility in a Sensor Network of Mobile Phones, NOSSDAV'07 Urbana, Illinois USA

[37] S. H.L. Liang, A. Croitoru, C. V. Tao, "A distributed geospatial infrastructure for Sensor Web", Computers \& Geosciences, 31, (2005), 221-231.

[38] S. H.L. Liang, C. Y. Huang, "GeoCENS: A Geospatial Cyberinfrastructure for the WorldWide Sensor Web", Sensors 2013, 13, 1340213424; doi:10.3390/s131013402.

1. 